\title{
64. EVOLUTION OF SHORT-PERIOD COMETARY ORBITS DUE TO CLOSE APPROACHES TO JUPITER
}

\author{
O. HAVNES \\ Astronomical Institute, Utrecht, The Netherlands*
}

\begin{abstract}
Evolution of short-period cometary orbits under the influence of close and moderately close approaches to Jupiter is studied. We have restricted the discussion to orbits in Jupiter's orbital plane and have neglected distant perturbations by Jupiter. The general evolution is a diffusion towards orbits of larger semimajor axes and a loss of comets, mainly due to ejection along hyperbolic orbits. We have found that the dynamical evolution of the orbits severely alters the assumed initial distribution of orbits during a time of some hundred years. We also tried to obtain an idea of the importance of close approaches as compared to the effect of moderate approaches to Jupiter for the evolution of the orbits. This was done by calculating the evolution of a group of comets twice, first considering the effect of perturbations up to a distance of 1.0 AU from Jupiter and then to a distance of $2.0 \mathrm{AU}$. The statistical results of the two calculations are in general agreement, indicating that close approaches to Jupiter mainly determine the evolution of the short-period comets.
\end{abstract}

\section{Introduction}

In any test of theories on the origin of comets, one of the major questions is whether or not the distribution of short-period comets can be satisfactorily reproduced. Numerical calculations on the orbits of known short-period comets over time intervals of $400 \mathrm{yr}$ (Kazimirchak-Polonskaya, 1967) show that these orbits are generally not stable and that their evolution seems to be conditioned by close approaches to Jupiter. It is therefore of interest to examine to what degree a group of short-period comets is affected by such repeated close approaches to Jupiter and on what time scale this effect is of importance.

\section{Method of Calculation and Results}

We have attempted to find general features of the evolution of short-period comets by combining the evolution of many different orbits. Each cometary orbit is calculated by regarding the comet as part of a three-body problem consisting of the Sun, Jupiter, and the comet. We consider the planar case only. Kazimirchak-Polonskaya's (1967) work indicated that close approaches to Jupiter were most important for the evolution of the cometary orbits. We therefore neglected distant perturbations from Jupiter.

Three groups of comets were studied in which the perturbations by Jupiter on the comet were calculated when their mutual distance was less than a limit $\Delta=1.0 \mathrm{AU}$. Two groups of comets in direct orbits were considered, the first group having semimajor axes from 3.0 to $8.0 \mathrm{AU}$, the second group from 3.1 to $8.1 \mathrm{AU}$, both in steps of $1.0 \mathrm{AU}$. We also considered a group of comets in retrograde orbits, having semimajor axes from 3.1 to 8.1 , also in steps of $1.0 \mathrm{AU}$.

* On leave from the Institute of Theoretical Astrophysics, University of Oslo, Blindern, Norway. 
The neglect of the more distant perturbations by Jupiter may introduce systematic errors in the statistical results. We therefore calculated the evolution of the first group of comets in direct orbits a second time where we included perturbations by Jupiter up to $\Delta=2.0 \mathrm{AU}$. The statistical results of the two calculations of different accuracy are in general agreement, indicating that the evolution of the short-period cometary orbits is mainly determined by close approaches to Jupiter.

The initial eccentricities $e_{0}$ which were considered are given in Table I as a function of initial semimajor axis $a_{0}$. For each pair of $a_{0}$ and $e_{0}$ we considered six different initial values of mean anomaly, varied in steps of $60^{\circ}$ from $0^{\circ}$ to $300^{\circ}$. Further details on the methods of numerical calculation and results for the cases when $\Delta=1.0 \mathrm{AU}$ are given in Havnes (1970).

TABLE I

Minimum initial eccentricity $e_{S}$ as a function of initial semimajor axis $a_{0}$

\begin{tabular}{|c|c|c|c|c|c|c|c|}
\hline & $a_{0}(\mathrm{AU})$ & 3.0 & 4.0 & 5.0 & 6.0 & 7.0 & 8.0 \\
\hline Direct orbits & & 0.75 & 0.45 & 0.35 & 0.45 & 0.55 & 0.55 \\
\hline Retrograde orbits & & 0.75 & 0.25 & 0.15 & 0.25 & 0.35 & 0.35 \\
\hline
\end{tabular}

The eccentricities which are considered are from $e_{0}=0.95$ to $e_{0}=e_{s}$ in steps of 0.1 . The values of $e_{S}$ are chosen so that the orbits are such as could result from direct capture of parabolic comets (Havnes, 1970).

Each orbit is followed for a time interval of $1000 \mathrm{yr}$ or until the comet is removed from the group, either by attaining a hyperbolic orbit or - in some cases - colliding with the Sun or Jupiter. The evolution of the first group of comets in direct orbits is shown in Figure 1, where the corresponding elements $a$ and $e$ are plotted at time $t=$ 100,500 , and $1000 \mathrm{yr}$. The comets having $e_{0}=0.95$ are omitted as they have short lifetimes (Shtejns, 1959). For the remaining comets the effects of limited lifetime have not been taken into account.

The loss of comets, in percentages of the initial numbers (the comets having $e_{0}=$ 0.95 are not counted), are given in Table II for the different groups of comets. Figure 2 shows the loss of comets in more detail. We have given the percentage of comets removed from the system as a function of $e_{0}$ and $a_{0}$, where the figures are in terms of the number of comets in each group having the same $e_{0}$ and $a_{0}$. The loss seems to depend little on the initial eccentricity but generally decreases with initial semimajor

TABLE II

The percentage loss of comets in different groups

\begin{tabular}{lllll}
\hline Group of comets & $\Delta$ (AU) & $t=100$ & $t=500$ & $t=1000 \mathrm{yr}$ \\
\hline Direct group number 1 & 1.0 & 2 & 9 & 12 \\
Direct group number 1 & 2.0 & 1 & 7 & 14 \\
Direct group number 2 & 1.0 & 1 & 8 & 15 \\
Retrograde group & 1.0 & 1 & 5 & 11 \\
\hline
\end{tabular}




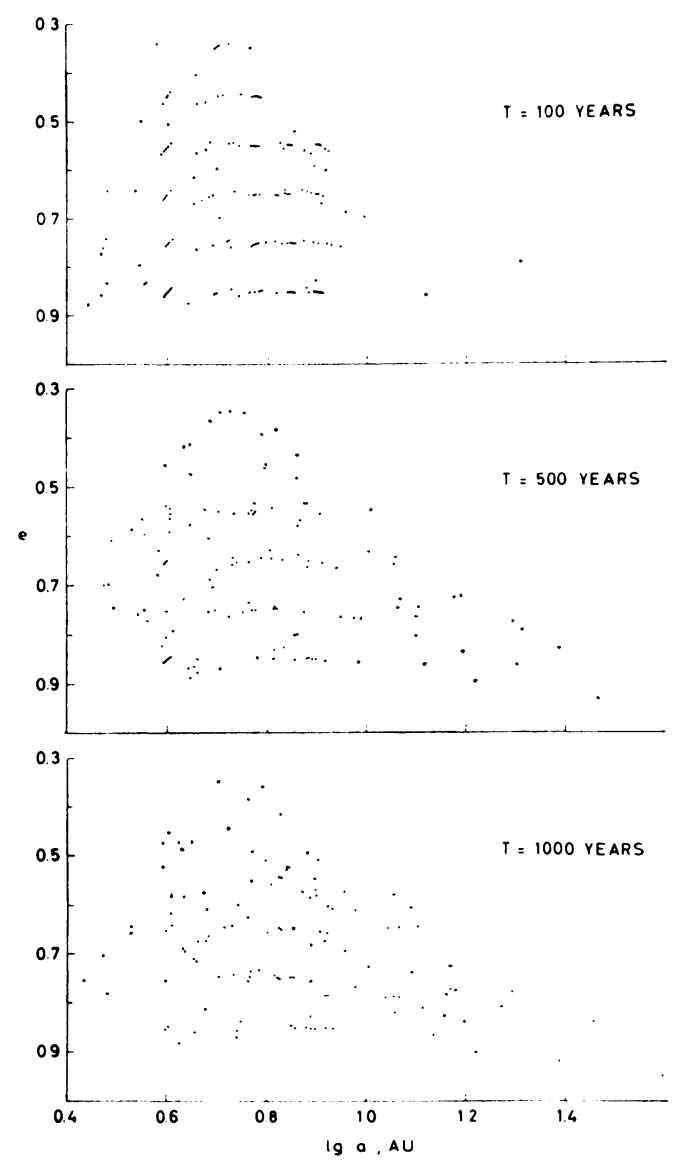

Fig. 1. The evolution of a group of short-period comets (orbiting in direct heliocentric orbits) due to close approaches to Jupiter is shown. The group of comets initially had semimajor axes in the interval 3.0 to 8.0 in steps of $1.0 \mathrm{AU}$. The corresponding initial eccentricities were varied from 0.85 in steps of 0.1 down to $e_{S}$ as given in Table I. The distribution of corresponding elements $a, e$ is shown at times $t=100,500$, and $1000 \mathrm{yr}$.

axis. This last effect is to be expected as comets with the largest periods (semimajor axes) generally have the smallest probability of passing close to Jupiter during a certain time interval.

The general evolution of a group of short-period comets - according to our calculations - is a steady loss of comets and a diffusion towards orbits having larger semimajor axes. This diffusion is apparent from Figure 1 for the first group of comets in direct orbits. In Table III we have demonstrated the diffusion for all the groups of comets by calculating the average of the reciprocal semimajor axis $\langle 1 / a\rangle$ at times $t=0,500$, and $1000 \mathrm{yr}$.

From Figure 2 it appears that the loss of comets in the retrograde group is generally less than for the corresponding direct groups. In Havnes (1970) it was stated that 'The rate of diffusion seems to be smaller for the retrograde comets than for the direct 


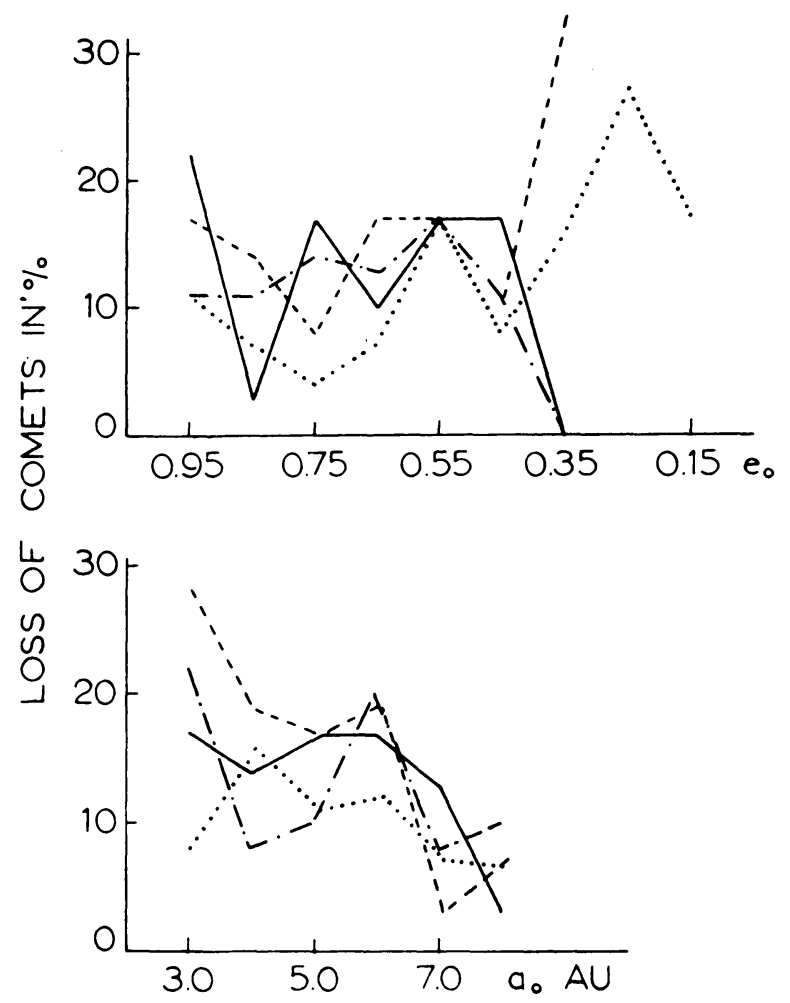

Fig. 2. The loss of comets (due to ejection from the system or collision with the Sun or Jupiter) for the different groups of comets as a function of initial eccentricity $e_{0}$ (upper figure) or initial semimajor axis $a_{0}$ (lower figure). The line ——- gives the results for the first group in direct orbits (3.0 $\mathrm{AU} \leq a_{0} \leq 8.0 \mathrm{AU}$ and $\Delta=1.0 \mathrm{AU}$ ) and -.. - . the results for the same group when $\Delta=2.0$ AU. For the second group in direct orbits $\left(3.1 \mathrm{AU} \leq a_{0} \leq 8.1 \mathrm{AU}\right.$ and $\left.\Delta=1.0 \mathrm{AU}\right)$ the results are given by -----, while $\cdots \cdots$ gives the results for the retrograde group $\left(3.1 \mathrm{AU}<a_{0} \leq 8.1 \mathrm{AU}\right.$ and $\Delta=1.0 \mathrm{AU}$ ). The large spread in the results for direct orbits of $e_{0}=0.35$ is caused by the small number ( 6 orbits) that in each case had initial eccentricity $e_{0}=0.35$.

TABLE III

The average of the reciprocal semimajor axes $\langle 1 / a\rangle$ for the different groups of comets

\begin{tabular}{lllll}
\hline Group of comets & $\Delta(\mathrm{AU})$ & $t=0$ & $t=500$ & $t=1000 \mathrm{yr}$ \\
\hline Direct group number 1 & 1.0 & 0.193 & 0.167 & 0.150 \\
Direct group number 1 & 2.0 & 0.193 & 0.172 & 0.143 \\
Direct group number 2 & 1.0 & 0.189 & 0.158 & 0.141 \\
Retrograde group & 1.0 & 0.185 & 0.155 & 0.132 \\
\hline
\end{tabular}

comets'. This statement resulted from a visual inspection of a plot for the retrograde comets similar to Figure 1. Here we find, according to Table III, that their rate of diffusion towards orbits of larger semimajor axes is somewhat larger than for the direct comets. However, the difference is small, and in view of this, we cannot explain 
the apparent nonexistence of retrograde comets in Jupiter's family as a result of a selection effect - differing between the direct and retrograde comets - in the evolution due to close approaches to Jupiter.

The effect of evolution on the distribution in semimajor axis of the first group of comets in direct orbits is shown in Figure 3. We have given the distribution for the two

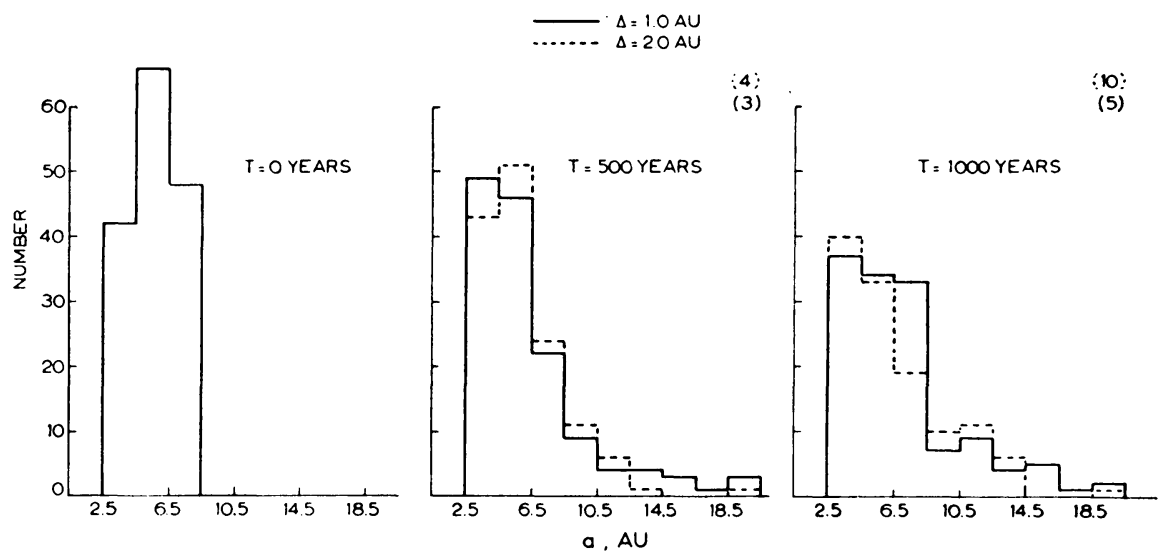

Fig. 3. The distribution of semimajor axis for the first group of comets in direct orbits is shown at $t=0,500$, and $1000 \mathrm{yr}$. The full and broken lines give the results by the calculations including perturbations out to distances of $\Delta=1.0$ and 2.0 AU, respectively, from Jupiter. The distributions are shown up to $a=20.5 \mathrm{AU}$. The number of comets having $a>20.5 \mathrm{AU}$ for the two groups are given in full parentheses and dotted parentheses, respectively.

calculations of different accuracy (perturbations by Jupiter on the comet for $\Delta=1.0$ and $2.0 \mathrm{AU}$, respectively) at times $t=0,500$, and $1000 \mathrm{yr}$. The distributions are in general agreement, indicating that the characteristics of the evolution of a group of short-period comets may be found by taking account of close approaches to Jupiter only.

\section{Conclusion}

Our calculations show that the distribution of a group of short-period comets changed considerably during a few hundred years. According to this, the evolution of the orbits due to close approaches to Jupiter should be taken into account when calculating theoretical distributions of such comets if their lifetimes are of this order. As we have considered the planar case only, the evolution of cometary orbits having $i \neq 0$ may be expected to be slower than for the case with $i=0$. However, as the inclinations of the short-period comets generally are small, calculations of the evolution of such a group should not give results differing considerably from our results.

The results obtained indicated that the evolution of the orbits is dominated by the effect of the close approaches to Jupiter. This conclusion was reached by comparing statistical results of two calculations of the evolution of a group of cometary orbits, taking into account perturbations by Jupiter on the comet out to mutual distances 
$\Delta=1.0$ and 2.0 AU, respectively. Even if the perturbations by Jupiter are taken into account at greater distances, we think that the close approaches will still determine the evolution of the short-period comets. Such approaches are fairly numerous, e.g., for the comets having $a_{0}=5.0 \mathrm{AU}$ and existing in bound orbits during the integration period of $1000 \mathrm{yr}$ an average of 1.2 approaches to Jupiter closer than $1.0 \mathrm{AU}$ occurred per $100 \mathrm{yr}$. On the other hand, for comets with large inclinations and thereby smaller probability of passing close to Jupiter, the perturbations by Jupiter at larger distances may be of importance.

\section{References}

Havnes, O.: 1970, Icarus 12, 331.

Kazimirchak-Polonskaya, E. I.: 1967, Astron. Zh. 44, 349.

Shtejns, K. A.: 1959, Astron. Zh. 36, 512.

\section{Discussion}

B. G. Marsden: What method did you use for calculating the perturbations?

$O$. Havnes: In the outer parts I used Cowell's method in jovicentric coordinates, while if the distance became less than $0.2 \mathrm{AU}$ I used the method of variation of the hyperbolic jovicentric elements. 